\title{
Review of Effectiveness of Physiotherapy Maneuvers and Modalities in Management of Knee Pain Due to Osteoarthritis
}

\author{
Sumreen Z. ${ }^{1}$ \\ Address for Correspondence: Physiotherapist, Department of Physiotherapy, Mayo Hospital Lahore, Pakistan
}

Background: Exercise and other rehabilitation strategies are not frequently prescribed, although they are recommended by all published guidelines. ${ }^{1}$ There is limited access and affordability to multiple pharmacological and surgical interventions for relief, which everybody can not manage.

Objectives: Establishing physiotherapy intervention, a preferable treatment.

Methodology: Reviewing 11 articles meeting inclusion criteria and aggregating their results.

Results: While studying comparison of preferred treatments among pharmacological and non pharmacological for pain of knee osteoarthritis, the results show $70 \%$ confidence level for later resulted that evidence based care for adults with knee pain, delivered by primary care physiotherapists and pharmacists, resulted in short term improvements in health outcomes, reduced use of ANSAID and high patient satisfaction, ${ }^{2}$ than with pharmacists and orthopedic surgeons alone. Regarding physiotherapy techniques exercise regimen and postural guidance seem to give $80-90 \%$ batter results than modalities.

Conclusions: Ways to promote high quality evidence that physiotherapy practice should be identified and evaluated, also therapeutic exercises show better results than modalities managing O.A knee.

Keywords: Disability osteoarthritis (O.A), Physiotherapy management O.A, Pain management knee O.A, O.A older people.

\section{Introduction}

Osteoarthritis of the knee (O.A Knee) is the most common type of osteoarthritis (OA), and its prevalence is rising in parallel with the increasing age of the population. The condition is associated with pain and inflammation of the joint capsule, impaired muscular stabilization, reduced range of motion, and functional disability. ${ }^{3}$

Studies have shown that in UK $31 \frac{1}{2}$ million people over age of 65 suffer from this condition. Many working hours are less because of effects of O.A.

Both men and women are affected but Jt distribution pattern is different.

Incidence $=70 \%$ above 65 years.

Frequency $=$ Most frequent Jt disorders in world.

Condition resulting from Trauma, Dislocation, infection, deformity, obesity, Hemophilia, Acromegaly, hyperthyroidism, Tabes Dorsalis, syringeomyelia and charcots Jts is SECONDARY TYPE while ideopathical condition is known as PRIMARY TYPE. Beside consequences of other conditions, factors like Heredity, Poor posture, aging, climate, hormonal imbalance, defective lubricating mechanism (on even arthritis) and crystals in jts also contribute.

Being most frequent in Primary care, physicians label Chronic knee pain as O.A and prescribe medication to relieve pain, but there are concerns about efficacy, safety, the cost of medication (NSAIDS particularly), therapeutic exercises and other primary therapy techniques too. Furthermore palliative medications do not deal with functional impairments. Improving function is best achieved by exercise, self management, intervention and postural awareness (if patients experience benefits from all that). Combining exercise and self management together with all other physical therapy maneuvers might enhance their separate effects. Taking conclusion from these effects best prescription plan designed will be much more significant. With passage of time people live longer, pattern of incidence change, safe, effective and efficient intervention that improve function can be delivered to people.

\section{Objectives}

1. To review the studies/ work done for treatment of O.A Knee and establishing a preferable management strategy.

2. Introducing physiotherapy a first line Therapy for included criteria being non-invasive, non-pharmacological, non-surgical and reasonably priced option with few side effects in concomitant treatment of O.A Knee.

3. Informing readers about patient's preference and satisfaction level with therapeutic intervention.

\section{Material and Methods}

Analysis of efficacy of different clinical interventions regarding conventional and community physiotherapy Techniques.

Research is performed from 1966 to 2009 studies done. 70 Abstracts were studied regarding conservative/non-pharmacological management of chronic knee pain, out of which 20 were considered only. 9 were again excluded not showing relevant age group. 11 meet inclusion criteria.

\section{Inclusion Criteria}

The articles having details of patients with established 
symptomatic O.A Knee unilateral / bilateral (following revised criteria of American College of Rheumatology).

- Conservative management plan designed by all health professionals.

- $\quad$ Age $>18$ years.

- Symptom duration at least more than 3 months.

- Areas.

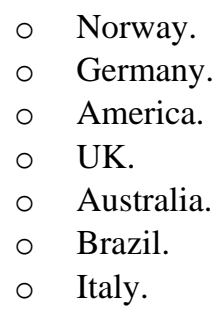

\section{Exclusion Criteria}

Articles studying patients with co-existing inflammatory jt diseases in serious co morbidities:

Pharmacological treatment by all health professionals.

Arthritis knee with acute inflammation / recent injuries. Scheduled knee replacement $<6$ months.

\section{Results}

Rehabilitated participants have get better functioning level than those having primary care. $\%$ age difference $=95: 5$.

TENS (Transcutaneous electrical nerve stimulation).

EA (Electro-acupuncture).

LLLT (Low level laser therapy).

All administered with optimal doses in an intensive 2-4 weeks $R_{x}$ regimen seen to offer clinically relevant shortterm pain relief for O.A knee. ${ }^{4}$

Patients self management/education program can significantly improve knowledge, compliance, behavior, and health outcome, however effectiveness differ between programs and disease states.

Results are seen within 4 weeks (short - term efficacy) to 12 weeks (pain relief at follow up) with different interventions variably.

\section{Discussion}

This reviews devised a comprehensive rehabilitation program integrating a simple, personalized, progressive exercise regimen, with patient education, self management, use of modalities, and active coping strategies enabling better managing arthritic knee pain. ${ }^{4}$ My primary hypothesis was comparison of different treatment strategies designed for arthritis (Uni and bilateral knee pain), and concluding the better plans to save time, money, and related resources just to finding out what will relieve a person if he / she is suffering form disabling knee pain.
A subsidiary hypothesis was underling the frequently used therapeutic techniques, modalities and the medication other than self management and postural guidance to have maximum knowledge about management plans regarding O.A knee.

Study has compared differential management plans for people with O.A in different communities.

Beside other techniques self management is one that aims to motivate people to undertake the changes in behavior necessary to improve their treatment. ${ }^{5}$ Disease specific self management program differs from other more generic arthritis. Programmed study evaluate use of variety of modalities including TENS, LLLT (low level laser therapy), Acupuncture, short wave diathermy, ultrasound, hot and cold packs (cryo therapy), massage, traction, glides, stretching, pulsed electromagnetic field, static magnets, braces and orthosis as treatment and preventive causes along with numerous open and closed chain exercises for effective, distal and proximal JTs for relief of pain and stability respectively.

The result of study will provide evidence to guide clinicians to establish priorities regarding provision of disease specific treatment plan. As people with O.A of knee have identified pain and problem with daily activities as much important problem associated with their condition. HENCE, the results of study will reflect most appropriate choice for them to get relief.

\section{Conclusion}

Studying efficacy of physical interventions, modalities, exercise regimens, medication, self management, patient education and postural guidance ${ }^{6-8}$ then compiling their effects in terms of percentage efficacy of each of technique relieving chronic knee pain in osteoarthritis. It is concluded that modalities give better results using adjuvant to opoids and non opoids + NSAIDS rather then using alone, in sub acute to chronic symptoms including inflammatory symptoms only. ${ }^{9}$ 
Also implementation of better self management programs, exercises and postural guidance, given better prevention and symptomatic relief than medication or modalities.

Most chronic knee pain is managed with medication despite concerns about safety, efficacy and cost, management guidelines recommendation and people's management preferences. Previous experience and perceptions of need were major determinants of people's preferences, but many people were unaware of management options. Appreciating patient preference and provision of more information about management options are important in facilitating informed patient / clinician discussion and agreement. ${ }^{10}$

This study provides information about physiotherapy performance in patients with knee O.A. Exercise is the most common treatment and this is supported by high quality evidence.

Most chronic knee pain is managed with medication despite concerns about safety, efficacy and cost, management guidelines recommendation and people's management preferences. Previous experience and perceptions of need were major determinants of people's preferences, but many people were unaware of management options. Appreciating patient preference and provision of more information about management options are important in facilitating informed patient / clinician discussion and agreement. ${ }^{10}$

This study provides information about physiotherapy performance in patients with knee O.A. Exercise is the most common treatment and this is supported by high quality evidence. Physiotherapists also provide several treatment modalities based on moderate and low quality evidence of benefit, or without evidence from systematic reviews. We need more research to develop and identify the best method to measure physiotherapy performance in primary care. ${ }^{11}$

\section{References}

1. Scarpa R, Sarzi-Puttini P, Cimmino MA, Caporali R, Parazzini F, Zaninelli A, Canesi B: Analysis of pharmacologic and nonpharmacologic prescription patterns of general practitioners and specialists in the AMICA study. Semin Arthritis Rheum. 2005 Aug; 35 (1 Suppl 1): 24-30.

2. Elaine M Hay, professor/consultant in community rheumatology, Nadine E Foster, DoH primary career scientist and senior lecturer in therapies, Elaine Thomas, senior lecturer in biostatistics, George Peat, senior lecturer in clinical epidemiology, Mike Phelan, medication review pharmacist, Hannah E Yates, research nurse, Alison Blenkinsopp, professor of the practice of pharmacy, and Julius Sim, professor of health care research: Effectiveness of community physiotherapy and enhanced pharmacy review for knee pain in people aged over 55 presenting to primary care : pragmatic randomized trial. BMJ. 2006 November 11; 333 (7576): 995.

3. Jan M Bjordal, Mark I Johnson, Rodrigo AB LopesMartins, Bard Bogen, Roberta Chow and Anne E Ljunggren : Short term efficacy of physical interventions in osteoarthritic knee pain. A systematic review and meta-analysis of randomized placebo-controlled trials. BMC Musculoskeletal Disorders 2007; 8: 51.

4. M. V. Hurley, N. E. Walsh, H. L. Mitchell, T. J. Pimm, A. Patel, E. Williamson, R. H. Jones, P. A. Dieppe, B. C. Reevies : Clinical Effectiveness of a Rehabilitation Program Integrating Exercise, Self-Management, and Active Coping Strategies for Chronic Knee Pain : A Cluster Randomized Trial. 2007, American College of Rheumatology.

5. S Coleman, NK Briffa, G Carroll, C Inderjeeth, N Cook and J McQuade: Effects of self - management, education and specific exercises, delivered by health professional, in patients with osteoarthritis of the knee. BMC Musculoskeletal Disorders 2008; 9: 133.

6. Gaker KR, Nelson ME, Felson DT, Layne JE, Sarno R, Roubenoff $R$ : The efficacy of home based progressive strength training in older adults with knee osteoarthritis: a randomized controlled trial. J Rheumatol. 2001 Jul; 28 (7): 1655-65.

7. Dias RC, Dias JM, Ramos LR: Impact of an exercise and walking protocol on quality of life for elderly people with OA of the knee. Physiother Res Int. 2003; 8 (3): 121-30.

8. McCarthy CJ, Mills PM, Pullen R, Richardson G, Hawkins N, Roberts CR, Silman AJ, Oldham JA: Supplementation of home-based exercise programme with a class - based programme for people with osteoarthritis of the knees: a randomized controlled trial and health economic analysis. Health Technol Assess. 2004 Nov; 8 (46) iii-iv: 1-61.

9. Martin Schencking, Adriane Otto, Tobias Deutsch and Hagen Sandholzer : A comparison of Kneipp hydrotherapy with conventional physiotherapy in the treatment of osteoarthritis of the hip or knee : protocol of a prospective randomized controlled clinical trial. BMC Musculokeletal Disorders 2009; 10: 104.

10. Helene L Mitchell and Michael V Hurley : Management of chronic knee pain: A survey of patient preferences and treatment received. BMC Musculoskeletal Disorder 2008; 9: 123.

11. Sarzi-Puttini P, Cimmino MA, Scarpa R, Caporali R, Parazzini F, Zaninelli A, Atzeni F, Marcolongo R: Do physicians treat symptomatic osteoarthritis patients properly? Results of the AMICA experience. Semin Arthritis Rheum. 2005 Aug; 35 (1 Suppl 1): 38-42. 